\title{
Impact of treatment completion, intolerance and adverse events on health system costs in a randomised trial of 4 months rifampin or 9 months isoniazid for latent TB
}

\author{
Anne Aspler, ${ }^{1,2}$ Richard Long, ${ }^{2}$ Anete Trajman, ${ }^{3}$ Marie-Josée Dion, ${ }^{1}$ Kamran Khan, ${ }^{4}$ \\ Kevin Schwartzman, ${ }^{1}$ Dick Menzies ${ }^{1}$
}

See Editorial, p 572

- Supplementary tables are published online. To view these files please visit the journal online (http://thorax.bmj.com).

${ }^{1}$ Respiratory Epidemiology and Clinical Research Unit, Montreal Chest Institute, McGill University, Canada

${ }^{2}$ Faculty of Medicine, University of Alberta, Canada

${ }^{3}$ Department of Medicine, Pró-Reitoria de Saúde, Gama Filho University, Brazil

${ }^{4}$ Department of Medicine, St Michael's Hospital, University of Toronto, Canada

Correspondence to Dr Dick Menzies, Montreal Chest Institute, 3650 St-Urbain, Room K1.24, Montreal, Quebec, Canada H2X 2P4;

dick.menzies@mcgill.ca

Received 7 August 2009 Accepted 26 March 2010
ABSTRACT

Rationale Treatment for latent tuberculosis infection with isoniazid for 9 months (9INH) has poor completion and serious adverse events, while treatment for 4 months with daily rifampin (4RIF) has significantly higher completion and fewer adverse events.

Objectives To compare the health system costs of 4RIF and $9 \mathrm{INH}$

Methods In a randomised trial conducted in five Canadian centres, one Brazilian and one Saudi Arabian centre, consenting subjects were randomised to receive $4 \mathrm{RIF}$ or $9 \mathrm{INH}$. Health system costs were estimated from healthcare utilisation including scheduled and unscheduled visits, investigations and drugs. All activities for all subjects were evaluated using financial information from 2007 from the Montreal Chest Institute. Costs were expressed in Canadian dollars.

Results Total health system cost per patient allocated to 4RIF was $\$ 854$ compared with $\$ 970$ for 9INH ( $p<0.0001$ ). The average cost per patient for the 328 of 420 (78\%) who completed 4RIF therapy was $\$ 1094$ compared with $\$ 1625$ for the 254 of $427(60 \%)$ completing 9INH $(p<0.0001)$. Costs were modestly increased in patients with minor intolerance and substantially increased if the treating physician stopped treatment because of possible adverse events. Total costs related to management of adverse events with 9INH were \$48 142 compared with \$25684 for 4 RIF $(p=0.008)$. Using these data, incremental costeffectiveness analyses showed that 4RIF would be cost saving and prevent more cases within 2 years if efficacy exceeded $74 \%$, and cost saving if efficacy exceeded $65 \%$. Conclusions The 4 RIF regimen was significantly cheaper per patient completing treatment because of better completion and fewer adverse events.

RCT registration number NCT00170209.

\section{INTRODUCTION}

Two billion of the world's population is estimated to have dormant or latent tuberculosis (TB) infection (LTBI). Of those infected, it is estimated that 9.2 million develop active TB each year, of whom 1.7 million die-the greatest cause of death from infectious disease after $\mathrm{HIV}^{1}{ }^{1}$ despite the existence of effective treatment for $\mathrm{LTBI}^{2}$

The currently recommended standard treatment for LTBI is a once-daily regimen of isoniazid taken for 9 months (9INH). ${ }^{2}{ }^{3}$ In the USA this is used to treat more than $80 \%{ }^{4}$ of the estimated 250000 persons ${ }^{56}$ who are prescribed LTBI treatment each year, as it has efficacy of $90 \%{ }^{7}$ if patients complete treatment. However, in routine practice, less than $50 \%$ of patients complete treatment, ${ }^{8}$ substantially reducing the effectiveness of this approach. Treatment for 4 months with daily rifampin (4RIF) is a recommended alternative ${ }^{2} 3$ for persons intolerant of INH or exposed to persons with INH-resistant TB. 4RIF has been shown to have significantly higher rates of completion and lower rates of hepatotoxicity than $9 \mathrm{INH}^{9-11}$ We have completed a multicentre randomised trial to compare the rate of serious adverse events, treatment completion and health system costs with $4 \mathrm{RIF}$ and $9 \mathrm{INH}$. The findings with regard to treatment completion and adverse events have been reported elsewhere. ${ }^{9}$ The comparison of costs and relationship of costs to these treatment outcomes is reported here.

\section{METHODS}

\section{Study design and data gathering}

An open-label randomised controlled trial was conducted at seven university-affiliated hospitals, five in Canada and one each in Saudi Arabia and Brazil. Eligible adults with a documented positive tuberculin skin test ${ }^{3}$ whose treating physician had recommended treatment for LTBI signed informed consent. All patients were considered eligible unless they had absolute contraindications to the use of INH or RIF, regardless of risk factors for adverse events or non-compliance, as long as their treating physician believed that treatment of LTBI was indicated.

We wished to ascertain study outcomes and costs under routine conditions, so patients were followed by their usual treating physician who made all management decisions. If treatment was stopped by the treating physician because of a possible adverse event, these were reviewed by a threemember independent panel blinded to the study drug. Treatment outcomes were classified as:

Completed: if they took at least $80 \%$ of doses within 180 days for 4RIF and 365 days for 9INH. This group was subdivided into those who reported symptoms during follow-up (poor tolerance) and those who did not.

Default: patients who refused treatment, dropped out or took less than $80 \%$ of doses. This group was also subdivided into those who reported symptoms and those without symptoms. 
Physician stopped treatment permanently: this group was classified by the independent review panel into grade $1-2$ or grade $3-4$ adverse events or not a drug-related adverse event.

\section{Measuring and defining costs}

We estimated direct costs from the perspective of the healthcare system. Healthcare utilisation of each study participant was recorded at all centres; these were divided into two categories of scheduled and non-scheduled care. Scheduled care included pretreatment and scheduled follow-up clinic visits, physician and pharmacist fees, nursing care, routine blood and radiological tests and TB medications. Non-scheduled care included walk-in clinic or emergency visits, treatment-related hospitalisations, additional nursing visits, investigations, speciality consultations and medications for adverse events. We did not include building or administration costs, patients' out-of-pocket expenses or their indirect costs related to time lost.

All activities (measured at all centres) were evaluated using financial information from 2006 to 2007 from the Montreal Chest Institute, Montreal, Canada. Physician and pharmacist activities and medication costs were based on reimbursement schedules of the Ministry of Health of Quebec effective in 2007 (see table S1 in online supplement). For secondary analyses, the same activities were assigned values using financial information obtained in July 2007 from the University Health Network Hospital, Toronto, Canada (by KK) and from the Brazilian Ministry of Health, Brazil (by AT) (see tables S2 and S3 in online supplement summarising the most important personnel, laboratory and medication costs for each site. Brazil costs were converted to $\$ \mathrm{CAD}$ : $\$ 1.00 \mathrm{CAD}=1.73$ Real). ${ }^{12}$ In a separate analysis we varied only the cost of drugs based on the prices set by the Global Drug Facility. ${ }^{13}$ This facility provides high-quality TB drugs, yet the prices for rifampin and isoniazid are $97 \%$ and $87 \%$ lower, respectively, than in Canadian pharmacies.

\section{Cost comparison analysis}

Costs were calculated as the product of the unit cost for each activity and the frequency of that activity. A 1-year analytical horizon was used, corresponding to the maximum time of treatment, so costs were not discounted. ${ }^{14} 15$ Differences in costs between treatment groups (4RIF and 9INH) were tested for significance using Student $t$ tests or ANOVA for normally distributed costs, otherwise Wilcoxon rank sum tests were used. ${ }^{16}$ We estimated average treatment costs for each arm per patient randomised and per patient who completed treatment. Costs per month of treatment were calculated based on the total number of person-months of treatment.

All data were analysed using Stata Version 9.2 (Stata Corp, College Station, Texas, USA).

\section{Cost-effectiveness analysis}

We calculated an incremental cost-effectiveness ratio for treatment of newly infected close contacts with the two regimens using a 2 -year analytical horizon. We assumed that $5 \%$ of these contacts would develop active TB over the next 2 years if untreated, ${ }^{17} 18$ and that 9 months of treatment with isoniazid would have $90 \%$ efficacy $^{7}$ if completed properly. This meant that $0.5 \%$ of those completing $9 \mathrm{INH}$ (and $5 \%$ of those not completing 9INH) would develop active TB over the 2 years of the analysis. We also assumed that all patients were infected with strains that were sensitive to isoniazid and rifampin. The efficacy of 4RIF is presently unknown. In the base case analysis we assumed efficacy would be the same as $9 \mathrm{INH}(90 \%)$, but in sensitivity analysis varied this to as little as $60 \%$. This minimum was selected because, in a randomised trial, 3 months of treatment with rifampin had efficacy of $63 \%$ in preventing active TB among subjects with LTBI and silicosis. ${ }^{19}$ We calculated the expected cases over 2 years based on these efficacy assumptions and the observed completion of each regimen in the trial. The difference between the number of cases expected with each type of treatment and the number expected without any treatment was the estimated number of prevented cases within 2 years. The total costs observed in the trial were divided by this number to estimate the cost per case prevented for each regimen. We performed a two-way sensitivity analysis comparing both the cost and efficacy of 4RIF relative to $9 \mathrm{INH}$ in order to assess the robustness of the incremental cost-effectiveness ratio (ICER). ${ }^{14}$ In this analysis we repeated the ICER calculation as we increased the total cost per patient on 4 RIF in $5 \%$ increments from the observed value to $25 \%$ higher. At each of these cost values we estimated the ICER while decreasing in $5 \%$ decrements 4 RIF efficacy through a range from $90 \%$ to $60 \%$

\section{RESULTS}

Between 27 April 2004 and 31 January 2007, treating physicians at participating centres recommended LTBI therapy to 1008 eligible patients. The results regarding patient characteristics and the outcomes of adherence and severe adverse events are reported elsewhere. ${ }^{9}$ In brief, 60 screened patients were ineligible, 161 declined to participate and 847 were randomised. All baseline characteristics were similar in participants randomly assigned to the two regimens. Of the 420 randomised to 4RIF, $328(78 \%)$ completed treatment compared with $255(60 \%)$ of 427 patients allocated to 9INH. Grade 3-4 adverse events occurred in 17 (4\%) of those randomised to 9INH compared with $7(1.7 \%)$ of the 4RIF group $(p=0.04)$. Grade $3-4$ hepatotoxicity occurred in $16(3.8 \%)$ vs $3(0.7 \%)$ of those taking 9 INH and 4RIF, respectively $(\mathrm{p}=0.003){ }^{9}$

\section{Detailed healthcare utilisation and costs}

As shown in table 1, total costs for 4RIF were $\$ 358690$ and for 9INH were $\$ 414280$. The largest components of costs for 4RIF were routine clinic visits (38\%), drugs and pharmacy fees $(30 \%)$, routine blood tests $(7 \%)$ and non-scheduled care for evaluation and management of suspected adverse events (7\%). For 9INH the different components accounted for different proportions: routine visits accounted for $57 \%$, drugs and pharmacy charges $12 \%$, routine testing $7 \%$ and unscheduled visits $12 \%$ of total costs. The total cost per patient allocated to 4RIF was $\$ 116$ less than the total cost per patient allocated to 9INH. This reflected significantly lower average costs for scheduled and unscheduled care with 4RIF. The differences in cost between the two regimens were even greater when expressed per patient who completed treatment, reflecting the significantly better treatment completion with 4RIF. In secondary analyses, using health system costs from Toronto, total treatment costs of both regimens were very similar to Montreal treatment costs; when using Brazil health system costs, both regimens were much cheaper (4RIF: $\$ 323$ vs 9INH: $\$ 425, p<0.0001$ ). In both of these analyses, 4RIF remained the cheaper regimen (data not shown in tabular form). When the analysis was repeated using Montreal health system costs but the TB drug prices from the Global Drug Facility, 4RIF would have cost $\$ 658$ per patient randomised compared with $\$ 934$ for 9INH $(p<0.0001)$.

Table 2 summarises the relationship between symptoms, treatment completion and costs. Of 328 patients completing 
Table 1 Detailed healthcare utilisation and total costs of treatment with rifampin for 4 months (4RIF) vs isoniazid for 9 months (9INH) (all costs in Canadian \$)

\begin{tabular}{|c|c|c|c|c|c|c|c|}
\hline & 4RIF & & & 9INH & & & \\
\hline & Total cost & $\begin{array}{l}\text { Total per } \\
\text { patient } \\
\text { allocated }\end{array}$ & $\begin{array}{l}\text { Total per } \\
\text { patient } \\
\text { completed }\end{array}$ & Total cost & $\begin{array}{l}\text { Total per } \\
\text { patient } \\
\text { allocated }\end{array}$ & $\begin{array}{l}\text { Total per } \\
\text { patient } \\
\text { completed }\end{array}$ & p Value* \\
\hline Clinical visits & & & & & & & \\
\hline Baseline & $\$ 68334$ & & & $\$ 69473$ & & & \\
\hline$M D \pm R N$ follow-up & $\$ 94177$ & & & $\$ 152842$ & & & \\
\hline Medication & & & & & & & \\
\hline Pharmacy fees & $\$ 10373$ & & & $\$ 20251$ & & & \\
\hline Drugs for LTBI & $\$ 94825$ & & & $\$ 25609$ & & & \\
\hline Subtotal treatment of LTBI & $\$ 105198$ & $\$ 250$ & $\$ 321$ & $\$ 45860$ & $\$ 107$ & $\$ 180$ & \\
\hline Investigations & & & & & & & \\
\hline Complete blood count (CBC) & $\$ 11628$ & & & $\$ 11277$ & & & \\
\hline Non-scheduled care & & & & & & & \\
\hline Clinical visits & & & & & & & \\
\hline Emergency room visit & $\$ 179$ & & & $\$ 894$ & & & \\
\hline Unscheduled clinic visit & $\$ 701$ & & & $\$ 1051$ & & & \\
\hline Specialist consultation & $\$ 2937$ & & & $\$ 3247$ & & & \\
\hline Visit to evaluate adverse events & $\$ 9415$ & & & $\$ 18691$ & & & \\
\hline Telephone call & $\$ 46$ & & & $\$ 162$ & & & \\
\hline Visit to perform blood tests & $\$ 725$ & & & $\$ 1670$ & & & \\
\hline Subtotal clinical visits & $\$ 14003$ & $\$ 33$ & $\$ 43$ & $\$ 25715$ & $\$ 60$ & $\$ 101$ & \\
\hline Subtotal treatment for side effects & $\$ 218$ & $\$ 1$ & $\$ 1$ & $\$ 226$ & $\$ 1$ & $\$ 1$ & \\
\hline Investigations & & & & & & & \\
\hline Pulmonary function tests & $\$ 352$ & & & $\$ 440$ & & & \\
\hline Procedures & $\$ 1027$ & & & $\$ 546$ & & & \\
\hline Imaging & $\$ 50$ & & & $\$ 369$ & & & \\
\hline Subtotal investigations & $\$ 11464$ & $\$ 27$ & $\$ 35$ & $\$ 22201$ & $\$ 52$ & $\$ 87$ & \\
\hline Subtotal: non-scheduled care & $\$ 25685$ & $\$ 61$ & $\$ 79$ & $\$ 48142$ & $\$ 113$ & $\$ 189$ & 0.008 \\
\hline Overall total costs & $\$ 358690$ & 854 & 1094 & $\$ 414280$ & $\$ 970$ & $\$ 1625$ & $<0.0001$ \\
\hline
\end{tabular}

4RIF, 148 (45\%) reported some symptoms during treatment phase follow-up compared with 17 of 68 (25\%) who defaulted. The cost per patient and cost per patient-month were slightly higher in those with symptoms than in patients without symptoms, both in those who completed and those who defaulted. Among the 255 who completed 9INH, 136 (53\%) reported some symptoms during treatment compared with 48 $(36 \%)$ of the 133 who defaulted from 9INH. Costs per patientmonth were slightly higher in those with symptoms than in those without symptoms in each of these categories.

The treating physician was sufficiently concerned about the possibility of an adverse event to permanently stop the study drug in 41 subjects receiving $9 \mathrm{INH}$ and 28 subjects receiving
4RIF (table 3). Total costs per subject with suspected adverse events ranged from $\$ 1215$ for one patient with a drug interaction and $\$ 1143$ for each of the 28 patients with hepatotoxic reactions to $\$ 554$ per patient with gastrointestinal intolerance. Grade 3-4 serious adverse events were associated with average costs that were almost twice as high as the average cost for subjects with grade 1-2 adverse events. Using the results from table 3 and table S4 in the online supplement, unscheduled costs averaged $\$ 36$ in patients who did not report any symptoms during follow-up, $\$ 65$ in patients who reported minor symptoms, $\$ 366$ in patients with grade $1-2$ adverse events and $\$ 778$ in patients with grade $3-4$ adverse events. Costs for evaluation and management of specific adverse events averaged $\$ 1249$ for 
Table 2 Association of symptoms, adverse events and completion with costs (all costs in Canadian \$)

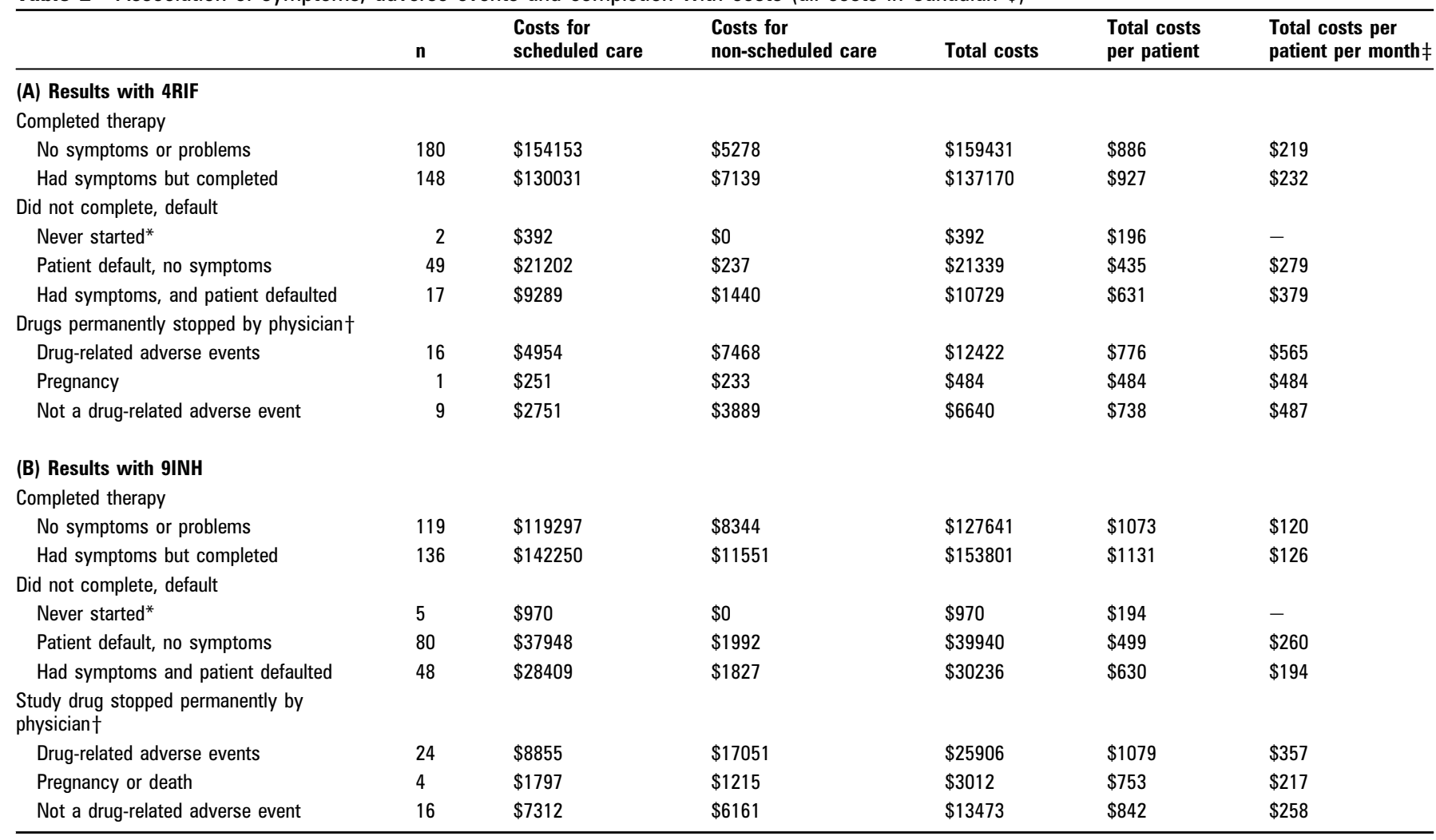

*Patients consented, were randomised, but then refused to start treatment. Costs are for baseline evaluation only.

†Final designation regarding whether drugs were stopped appropriately and severity/relationship to study drug were made by an independent panel, blinded to study drug. $\ddagger$ Patient-months calculated from total number (sum) of patient-months on treatment within each group.

9INH, isoniazid treatment for 9 months; 4RIF, rifampin treatment for 4 months.

grade 3-4 adverse events compared with $\$ 668$ for grade $1-2$ adverse events (see table S4 in online supplement).

\section{Cost-effectiveness analyses}

As shown in figure 1, using the observed completion rates and costs for each regimen, the cost per case prevented within 2 years with 4RIF was substantially less than the cost per case prevented with 9INH if the efficacy of 4RIF was the same as 9INH. This difference in costs per case prevented between the regimens would decline if 4 RIF had lower efficacy or higher costs. However, if 4RIF efficacy exceeds $75 \%$, this would still prevent more cases and provide net cost savings compared with 9INH. If the efficacy of 4 RIF was between $65 \%$ and $75 \%$, then 4RIF would be cheaper but prevent fewer cases. 9INH would prevent more cases and would be less expensive only if the efficacy of 4RIF was below the threshold of $65 \%$.

\section{DISCUSSION}

In this randomised trial, treatment of LTBI resulted in significantly lower health system costs per patient allocated to 4RIF than per patient allocated to 9INH. This was because of shorter treatment and fewer adverse events. The difference in cost per patient completing treatment was even greater because $78 \%$ completed 4RIF compared with only $60 \%$ completing 9INH. The efficacy of 4RIF is still undefined; however, if efficacy exceeds $65 \%$, corresponding to the efficacy documented with 3 months' treatment with rifampin in one randomised trial, ${ }^{19}$ then the regimen will be cost saving relative to $9 \mathrm{INH}$. We would expect these cost savings to be generalisable given that completion rates in the trial were similar to those seen under normal practice conditions in other programmes $\left(72 \%,{ }^{10} 74 \%^{20}\right.$ and $81 \%{ }^{11}$ with 4 RIF and $53 \%$ with $9 \mathrm{INH}^{10}{ }^{11}$ )

Findings with $9 \mathrm{INH}$ in this trial reflect the major limitations of current LTBI treatment. 9INH is considered the regimen of first choice for LTBI because of its high efficacy. ${ }^{7}$ However, in our trial and reports from several large programmes, ${ }^{10} 1121$ only $50-60 \%$ of patients who started $9 \mathrm{INH}$ completed the treatment, reducing the effectiveness of treatment to $50 \%$ or less. Although the medication is inexpensive, total costs for 9INH are high because close monitoring is required owing to the risk of drug-induced hepatitis. In this trial, hepatotoxicity accounted for $40 \%$ of patients whose treatment was permanently discontinued by their treating physicians and $57 \%$ of all non-scheduled costs in this group. Interestingly, almost one-third of patients in whom treatment was stopped for suspected INH hepatotoxicity were judged not to have this problem by the independent panel which was blinded to study drug. This reflects the heightened awareness and concern of treating physicians about INH hepatotoxicity. $^{22}$

The significantly lower rate of hepatotoxicity with 4RIF is therefore a very important potential advantage, especially if this finding is confirmed with broader clinical experience. To date, rifampin monotherapy has been associated with a very low rate of hepatotoxicity among elderly Chinese men, ${ }^{19}$ homeless persons in Boston, ${ }^{23}$ adolescents in California ${ }^{24}$ and a broad spectrum of patients in New Jersey ${ }^{11}$ and Maryland. ${ }^{10}$ Two important advantages of 4RIF-better completion and lower hepatotoxicity-therefore appear generalisable and should result in consistently lower costs for 4RIF in all settings. This experience is in marked contrast to experience with the 2 -month rifampin-pyrazinamide regimen in which completion was only slightly better ${ }^{25}$ 
Table 3 Costs for patients in whom drugs were permanently discontinued because of suspected adverse events (all costs in Canadian \$)

\begin{tabular}{|c|c|c|c|c|c|c|}
\hline & $\mathbf{n}$ & $\begin{array}{l}\text { Costs for } \\
\text { scheduled care }\end{array}$ & $\begin{array}{l}\text { Costs for } \\
\text { non-scheduled care }\end{array}$ & Total costs & $\begin{array}{l}\text { Average cost } \\
\text { per patient }\end{array}$ & p Value* \\
\hline \multicolumn{7}{|l|}{ Treatment regimen } \\
\hline 4RIF & 28 & $\$ 9667$ & $\$ 12232$ & $\$ 21899$ & $\$ 782$ & 0.05 \\
\hline 9INH & 41 & $\$ 15013$ & $\$ 24651$ & $\$ 39664$ & $\$ 967$ & \\
\hline \multicolumn{7}{|l|}{ Type of adverse event } \\
\hline Hepatotoxicity & 28 & $\$ 11063$ & $\$ 20942$ & $\$ 32005$ & $\$ 1143$ & $<0.001$ \\
\hline Haematological & 4 & $\$ 1271$ & $\$ 2694$ & $\$ 3965$ & $\$ 991$ & \\
\hline Rash & 17 & $\$ 5219$ & $\$ 6230$ & $\$ 11449$ & $\$ 673$ & \\
\hline Gastrointestinal intolerance & 7 & $\$ 1543$ & $\$ 2269$ & $\$ 3812$ & $\$ 545$ & \\
\hline Drug interaction & 1 & $\$ 305$ & $\$ 911$ & $\$ 1216$ & $\$ 1216$ & \\
\hline Other $\ddagger$ & 12 & $\$ 5280$ & $\$ 3837$ & $\$ 9117$ & $\$ 760$ & \\
\hline \multicolumn{7}{|l|}{ Final designation $†$} \\
\hline Grade 3-4 drug-related adverse event & 24 & $\$ 9317$ & $\$ 18668$ & $\$ 27985$ & $\$ 1166$ & $<0.001$ \\
\hline Grade $1-2$ drug-related adverse event & 16 & $\$ 4493$ & $\$ 5850$ & $\$ 10343$ & $\$ 646$ & \\
\hline Not a drug-related adverse event & 29 & $\$ 10870$ & $\$ 12364$ & $\$ 23234$ & $\$ 801$ & \\
\hline All patients in whom drugs were stopped & 69 & $\$ 24680$ & $\$ 36883$ & $\$ 61563$ & $\$ 892$ & - \\
\hline
\end{tabular}

*p Value from one-way ANOVA for comparison of mean costs within subgroups.

†Final designation regarding severity and relationship to study drug were made by an independent panel, blinded to study drug.

$\neq$ Other reasons for referral to independent review panel were: pregnancy $(n=5$ of whom 1 later completed therapy), arthralgia $(n=1)$, rheumatoid arthritis ( $n=1)$, fatigue ( $n=1)$, decreased libido $(n=1)$, dengue fever $(n=1)$, depression $(n=1)$ and death $(n=1)$. None of these was judged to be related to the study drug.

$9 \mathrm{NHH}$, isoniazid treatment for 9 months; 4RIF, rifampin treatment for 4 months.

and costs were significantly higher than with 6-9 months of $\mathrm{INH}^{2627}$ owing to the greater toxicity and consequent need for closer follow-up with the 2 -month regimen. ${ }^{2627}$ The advantage of lower hepatotoxicity with rifampin monotherapy would be lost if LTBI is treated with both isoniazid and rifampin for 3-4 months, as advocated by some. ${ }^{28}$

This study had a number of strengths. Most importantly, it was based on a randomised trial, ensuring balance of patient, health provider and centre characteristics that may profoundly influence costs. In contrast to many previous cost-effectiveness studies, no assumptions were made about care utilisation or outcomes. All outcomes were carefully ascertained in 847 subjects and costs were based on actual healthcare utilisation during follow-up. These patients were cared for by many providers in nine very different settings, enhancing generalisability of findings. The finding that 4RIF was significantly cheaper was true in all settings and did not change if we evaluated activities in different Canadian or Brazilian centres, used Canadian or international drug prices, or assumed a wide range of plausible efficacy. This provides evidence of the robustness of the findings.

Nevertheless, our study had limitations. Costs may have been overestimated due to the more intensive follow-up and greater attention to potential side effects. This is an inherent problem in any randomised trial, but should not have been different between the two regimens. We assumed that all latent infection was with pan-sensitive TB strains, overestimating the effectiveness of both regimens. However, this assumption would have overestimated the effectiveness of isoniazid to a greater extent than rifampin because initial isoniazid resistance is more common than rifampin resistance in the USA, ${ }^{29} \mathrm{Canada}^{30}$ and many other countries. ${ }^{31}$ An earlier analysis found that costeffectiveness of 4RIF would increase relative to $9 \mathrm{INH}$ with a higher prevalence of isoniazid resistance. ${ }^{32}$ Finally, patients' out-of-pocket expenses and time lost were not included in this analysis. However, these costs should have been higher for patients taking 9INH, given the significantly greater number of visits observed.
One potential risk of 4RIF is the creation of rifampin monoresistance. A recent meta-analysis ${ }^{33}$ concluded that the risk of isoniazid resistance was modestly increased by isoniazid treatment of LTBI. Although this may simply reflect inadvertent monotherapy of unrecognised active $\mathrm{TB}$, this risk is important because of the serious therapeutic implications of acquired rifampin resistance. ${ }^{34}$ However, rifampin mono-resistance following 4RIF has not been reported in trials, ${ }^{19}$ programme reports, ${ }^{10}{ }^{11}$ case series ${ }^{23} 24$ or in surveillance reports even though 4RIF is used to treat approximately $4 \%$ of all patients with LTBI in the USA. ${ }^{4}$ Nevertheless, it is important to maintain careful surveillance for the occurrence of this complication under routine programme conditions or in randomised trials.

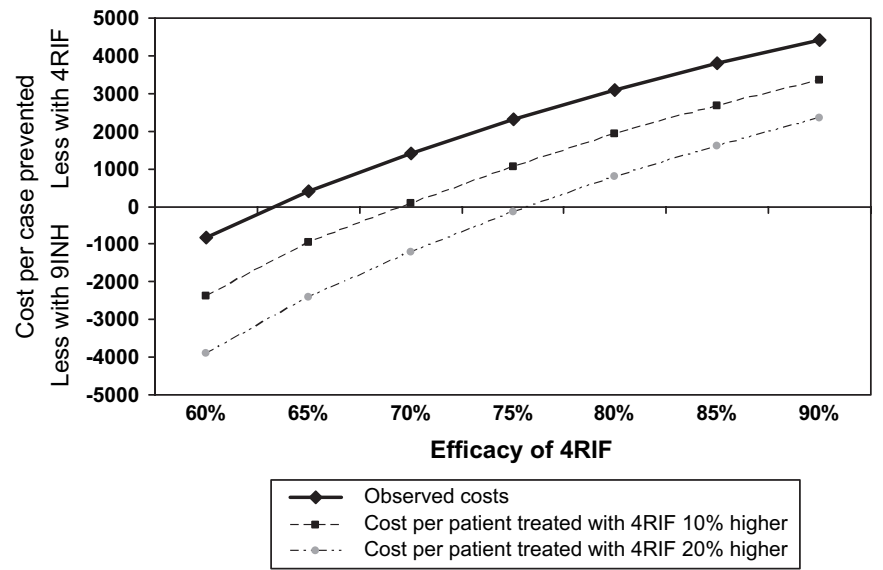

Figure 1 Difference in estimated cost per case prevented between rifampin taken for 4 months (4RIF) and isoniazid taken for 9 months (9INH). A value greater than zero means that the cost per case prevented with 4RIF was less than the cost per case prevented with 9INH. Solid line with diamonds represents analysis using observed costs with 4RIF and 9INH. Dashed line with squares represents total costs per patient randomised to $4 \mathrm{RIF} 10 \%$ more expensive than in base case analysis. Dashed and dotted line with circles represents total costs per patient randomised to 4 RIF $20 \%$ more expensive than in base case analysis. 
Our results may inform efforts for TB prevention. In the USA it has been estimated that 290000-433000 persons start treatment for LTBI, ${ }^{6}{ }^{6}$ of whom more than $80 \%$ take $9 \mathrm{INH}^{4}$ Based on estimates from this study, if all persons now taking 9INH were given 4RIF, this could result in savings of $\$ 22-33$ million, even without considering the benefit of better completion. Potential savings from a switch to 4RIF would also be substantial in Canada, given that more than $\$ 25$ million is spent annually on LTBI management. ${ }^{35}$ The medication cost for rifampin in Canada and in the USA is many times higher than the costs of the Global Drug Facility. ${ }^{12}$ If the same qualityassured rifampin ${ }^{12}$ was available in the USA, savings with use of 4RIF could be as much as \$66-98 million.

Better safety and improved completion rates are important reasons to consider the expanded use of the 4RIF regimen. Our study adds the advantage of significantly lower costs. We predict this regimen will be cost-effective if efficacy exceeds $65 \%$. A multicentre trial involving over 6000 subjects in seven countries to assess the efficacy of 4RIF is now underway.

Acknowledgements The authors are deeply indebted to (the late) Dr George Comstock, Dr Kevin Elwood, Dr Richard O'Brien and Dr Michael Lauzardo for serving as the independent review panel, and the Data Safety Monitoring Board for this study. The authors thank Dr Eric Rousseau and his team at the University of Sherbrooke for developing the web-based registration and randomisation software, as well as Ria Choe and Sara Korhani for assistance with manuscript preparation. The authors also thank the staff of the many TB clinics involved.

Funding This study was funded by a grant from the Canadian Institutes of Health Research (CIHR Grant Number MCT\#44154). DM currently holds a Chercheur National salary award, KS holds a Chercheur Boursier Clinicien Award from the Fonds de Recherche en Sante de Quebec (FRSO), AT receives salary support from ICOHRTA AIDS/TB FIC/NIH \# 5U2 R TW006883-03 and from FAPERJ (E-26/102.712/2008). None of these funding agencies had any role in the design, conduct, analysis or reporting of the results.

\section{Competing interest None.}

Ethics approval This study was conducted with the approval of the ethics committee of McGill University Health Centre and approved by institutional review boards in each participating institution.

Provenance and peer review Not commissioned; externally peer reviewed.

\section{REFERENCES}

1. World Health Organization. Global tuberculosis control. surveillance, planning, financing: 2008. (WHO/HTM/TB/2006.362). Geneva: World Health Organization, 2008

2. American Thoracic Society. Targeted tuberculin testing and treatment of latent tuberculosis infection. Am J Respir Crit Care Med 2000:161:S221-47.

3. Long RL, Ellis E. Canadian tuberculosis standards. 6th edn. Toronto: Canadian Lung Association, Public Health Agency of Canada, Tuberculosis Prevention and Control, 2007.

4. Horsburgh CR, Goldberg S, Bethel J, et al. Studies Consortium. Low latent tuberculosis infection treatment completion with the 9 month INH regimen. $\mathrm{Am} \mathrm{J}$ Respir Crit Care Med 2007;175:A24

5. Sterling TR, Bethel J, Goldberg S, et al. The scope and impact of treatment of latent tuberculosis infection in the United States and Canada. Am J Respir Crit Care Med 2006;173:927-31

6. McElroy PD, ljaz K, Lambert LA, et al. National survey to measure rates of liver injury, hospitalization, and death associated with rifampin and pyrazinamide for latent tuberculosis infection. Clin Infect Dis 2005;41:1125-33.

7. Comstock GM. How much isoniazid is needed for prevention of tuberculosis in immunocompetent adults. Int J Tuberc Lung Dis 1999;3:847-50.
8. Jasmer RM, Nahid P, Hopewell PC. Latent tuberculosis infection. $N$ Engl J Med 2002;347:1860-6.

9. Menzies D, Long R, Trajman A, et al. Adverse events with 4 months rifampin or 9 months isoniazid as therapy for latent TB infection: results of a randomised trial. Ann Intern Med 2008;149:689-97.

10. Page KR, Sifakis F, Montes de OR, et al. Improved adherence and less toxicity with rifampin vs isoniazid for treatment of latent tuberculosis: a retrospective study. Arch Intern Med 2006;166:1863-70.

11. Lardizabal A, Passannante M, Kojakali F, et al. Enhancement of treatment completion for latent tuberculosis infection with 4 months of rifampin. Chest 2006;130:1712-17.

12. Global Drug Facility. First-line tuberculosis drugs and formulations currently supplied/to be supplied by the global TB drug facility. World Health Organization, 2003. http://stoptb.org/GDF/drugsupply/drugs.available.html (accessed 31 Jul 2008)

13. Bank of Canada. Exchange rates 2007, 2007. http://www.bank-banque-canada.ca/ pdf/nraa04.pdf (accessed 23 Jul 2007).

14. Drummond MF, O'Brien B, Stoddart GL, et al. Methods for the economic evaluation of health care programmes. 2nd edn. New York: Oxford University Press, 1997.

15. Weinstein MC, Siegel JE, Gold MR, et al. Recommendations of the panel on costeffectiveness in health and medicine. JAMA 1996;276:1253-8.

16. Dawson-Saunders B, Trapp RG. Basic and clinical biostatistics. Norwalk: Appleton and Lange, 1990.

17. Sutherland I. Recent studies in the epidemiology of tuberculosis, based on the risk of being infected with tubercle bacilli. Adv Tuberc Res 1976;19:1-63.

18. Sutherland I. The evolution of clinical tuberculosis in adolescents. Tubercle 1966;47:308

19. Hong Kong Chest Service Tuberculosis Research Centre MBMRC. A doubleblind placebo-controlled clinical trial of three antituberculosis chemoprophylaxis regimens in patients with silicosis in Hong Kong. Am Rev Respir Dis 1992;145:36-41.

20. Haley CA, Stephan S, Vossel LF, et al. Successful use of rifampicin for Hispanic foreign-born patients with latent tuberculosis infection. Int J Tuberc Lung Dis 2008:12:160-7.

21. Nolan CM, Goldberg SV, Buskin SE. Hepatoxicity associated with isoniazid preventive therapy: a 7-year survey from a public health tuberculosis clinic. JAMA 1999;281:1014-18.

22. Sorresso D, Mehta JB, Harvill LM, et al. Underutilization of isoniazid chemoprophylaxis in tuberculosis contacts 50 years of age and older: a prospective study. Chest 1995:108:706-11.

23. Polesky A, Farber HW, Gottlieb DJ, et al. Rifampin preventive therapy for tuberculosis in Boston's homeless. Am J Respir Crit Care Med 1996;154:1473-7.

24. Villarino ME, Ridzon R, Weismuller PC, et al. Rifampin preventive therapy for tuberculosis infection. Am J Respir Crit Care Med 1997;155:1735-1.

25. Gao XF, Wang L, Liu GJ, et al. Rifampicin plus pyrazinamide versus isoniazid for treating latent tuberculosis infection: a meta-analysis. Int J Tuberc Lung Dis 2006; 10:1080-90.

26. Jasmer RM, Saukkonen JJ, Blumberg HM, et al. Short-course rifampin and pyrazinamide compared with isoniazid for latent tuberculosis infection: a multicenter clinical trial. Ann Intern Med 2002;137:640-7.

27. Jasmer RM, Daley CL. Rifampin and pyrazinamide for treatment of latent tuberculosis infection. Am J Respir Crit Care Med 2003;167:809-12.

28. Omerod LP. Rifampicin and isoniazid prophylactic chemotherapy for tuberculosis. Arch Dis Child 1998:78:169-71.

29. Center for Disease Control and Prevention. Reported tuberculosis in the United States, 2006. Atlanta, GA: US Department of Health and Human Services, Public Health Service, 2007.

30. Ellis E, Medaglia A, Phypers M, et al. Tuberculosis: drug resistance in Canada, 2006: reported susceptibility results of the Canadian tuberculosis laboratory surveillance system. Health Canada, 2007.

31. World Health Organization. Anti-tuberculosis drug resistance in the world report no.4. WHO/HTM/TB/2008. 2008. Geneva: World Health Organization; http:// whqlibdoc.who.int/publications/2008

32. Khan K, Muennig P, Behta M, et al. Global drug-resistance patterns and the management of latent tuberculosis infection in immigrants to the United States. N Engl J Med 2002;347:1850-9.

33. Balcells ME. INH resistance after INH therapy. Emerg Infect Dis 2006;12:744-9

34. Caminero JA. Treatment of multidrug resistant tuberculosis: evidence and controversies. Int J Tuberc Lung Dis 2006;10:829-37.

35. Menzies D, Lewis M, Oxlade 0. Costs for tuberculosis care in Canada. Can J Public Health 2008;99:391-6. 